\title{
La Percepción de Apego con la Escuela Como un Factor Protector Para Conductas Antisociales en Escolares Chilenos
}

\section{Perceived School Bonding as a Protective Factor for Antisocial Behavior Among Chilean Students}

\author{
Jorge J. Varela \\ Universidad del Desarrollo \\ Javier Torres-Vallejos \\ Universidad Tecnológica de Chile INACAP y Universitat de Girona \\ Constanza González y Oriana García \\ Universidad del Desarrollo
}

\begin{abstract}
La escuela puede ser considerada un espacio vital de desarrollo humano e incluso un factor protector para el desarrollo de conductas de riesgo en adolescentes. El presente estudio buscó examinar la percepción de un mayor apego escolar como un factor protector de la influencia de pares en conductas antisociales en el contexto chileno. Se usó una muestra estratificada con afijación igual de 815 escolares de $7^{\circ}$ año básico a IV año medio provenientes de 15 establecimientos escolares seleccionados aleatoriamente de 5 diferentes NSE de la Región Metropolitana, 46,6\% mujeres, con una edad promedio de 15,11 años $D E=1,78$ ). Se utilizó una adaptación del instrumento Communities That Care Youth Survey, analizando el reporte de conductas antisociales en la escuela, relación con pares antisociales, consumo de drogas por parte de pares y apego con la escuela. Por medio de un análisis de regresión múltiple, se evidenció un efecto de interacción, en donde para aquellos estudiantes que perciben un mayor apego con la escuela, la influencia de los pares con conductas antisociales se ve moderada, en comparación con los estudiantes que tienen una percepción de bajo apego con la escuela. Este resultado provee evidencia sobre la importancia del apego con la escuela para prevenir conductas antisociales en adolescentes y para informar programas de prevención en contextos escolares.
\end{abstract}

Palabras clave: conductas antisociales, adolescentes, escuela, apego escolar, regresión múltiple

\begin{abstract}
Schools can be regarded as vital spaces for human development and even as factors that protect adolescents from developing risk behaviors. The present study sought to examine the perception of greater school attachment as a protective factor against peer influence in antisocial behaviors in the Chilean context. A stratified sample was used, with an equal allocation of 815 students from 7 th to 12 th grade enrolled in 15 schools belonging to 5 SES categories, selected randomly in the Metropolitan Region (46.6\% female, with an average age of 15.11 years; SD = 1.78). An adaptation of the Communities That Care Youth Survey was used to analyze reports of antisocial behaviors at school, relationships with antisocial peers, drug use by peers, and attachment to school. A multiple regression analysis revealed an interaction effect: for students who perceive more attachment to their school, the influence of peers with antisocial behaviors is moderated relative to those with a perception of low school attachment. This result provides evidence of the importance of school attachment in preventing antisocial behaviors in adolescents and informing prevention programs in school contexts.
\end{abstract}

Keywords: antisocial behavior, adolescents, school, school bonding, multiple regression

La escuela ocupa un lugar de gran importancia en la sociedad, siendo uno de los principales pilares de formación y espacio donde niños, niñas y jóvenes se desenvuelven gran parte de sus días, recibiendo de este ambiente múltiples influencias que impactan y moldean sus trayectorias de desarrollo (Moshman, 2005). Así, la escuela puede ser un espacio significativo para el desarrollo infanto-juvenil, brindando oportunidades de desarrollo positivo, a través de la participación e involucramiento en la vida escolar (Eccles \& Roeser, 2011; Roeser, Eccles \& Sameroff, 2000). Algunos estudios han evidenciado que el contexto

Jorge J. Varela, Constanza González y Oriana García, Facultad de Psicología, Universidad del Desarrollo, Santiago, Chile; Javier Torres-Vallejos, Universidad Tecnológica de Chile INACAP, Santiago, Chile, y Universitat de Girona, España.

Los autores agradecen a la Fundación Paz Ciudadana, a Nicole Eisenberg de la University of Washington y a la Fundación San Carlos de Maipo.

La correspondencia relativa a este artículo debe ser dirigida a Jorge J. Varela, Facultad de Psicología, Universidad del Desarrollo, Avda. Plaza 680, Las Condes, Santiago, Chile. E-mail: jovarela@udd.cl 
escolar, incluso, puede moderar el efecto negativo de diferentes factores de riesgo sobre el desarrollo de conductas antisociales y desadaptativas en jóvenes (Kim, Gilman, Hill \& Hawkins, 2016; Varela et al., 2019). Según esto, el presente estudio buscó examinar el rol protector de la percepción individual de un mayor apego con la escuela, como factor protector, y de la influencia de pares que presentan comportamientos antisociales, como un factor de riesgo, sobre el desarrollo a nivel individual de conductas antisociales en adolescentes de Santiago, Chile.

\section{Conductas Antisociales en Jóvenes}

Las conductas antisociales se definen como aquellos comportamientos que habitualmente infringen las normas de convivencia social y que están dirigidas contra el entorno — propiedades y personas - de manera que se transgreden reglas y expectativas sociales (de la Peña Fernández, 2010; Dishion \& Patterson, 2006; Farrington, 2005; dos Santos, Holanda, Meneses, Luengo \& Gomez-Fraguela). Según diversos autores, las conductas antisociales aparecen durante la infancia y adolescencia (Eleni \& Giotsa, 2018; Moffitt, 1993; dos Santos et al., 2019) y representan una amplia gama de acciones que varían en términos cuantitativos y cualitativos, tales como agresiones, hurtos, vandalismo, piromanía, entre otras (Hawkins, Catalano \& Miller, 1992; Hein \& Barrientos, 2004).

En el contexto escolar, las conductas antisociales pueden verse reflejadas en reacciones agresivas hacia los pares y profesores, destrucción del mobiliario, absentismo y huidas (Espelage et al., 2013; Garaigordobil Landazabal, 2005), y quienes las cometen pueden terminar siendo excluidos por sus pares y de la comunidad escolar en general (Plazas et al., 2010). De esta manera, estudios muestran que conductas antisociales no solo afectan al entorno del estudiante que comete la conducta antisocial, sino también a su propio desarrollo psicosocial, reportándose mayor vulnerabilidad ante otros factores de riesgo en estos sujetos (Dishion \& Patterson, 2016). A su vez, se ha visto que quienes se ven involucrados en comportamientos antisociales en edades tempranas y por tiempos prolongados tienden a continuar con estas conductas y con mayor gravedad durante la adultez (Dishion \& Patterson, 2016; Farrington, 1995, 2005; Sanabria \& Uribe Rodríguez, 2009). Por lo mismo, resulta muy importante estudiar factores de riesgo y protección de este tipo de conductas en niños, niñas y adolescentes, que permitan reconocer con tiempo variables clave en ámbitos de prevención.

\section{Conductas Antisociales e Influencia de los Pares}

Desde una mirada ecológica, el desarrollo humano se entiende desde la interacción entre diferentes sistemas que influyen en el individuo (Bronfenbrenner, 1994; Eccles \& Roeser, 2011), en los que es posible identificar factores de riesgo y de protección que inciden en el comportamiento de los sujetos. Un factor de riesgo corresponde a características, variables o eventos que aumentan la probabilidad de que un individuo exprese distintos problemas de comportamiento, emocionales y del desarrollo (Fergus \& Zimmerman, 2005; Hein \& Barrientos, 2004; Sanabria \& Uribe Rodríguez, 2010).

De acuerdo a esto, existen diferentes predictores significativos de las conductas antisociales (Farrington, 2005; Sprague \& Walker, 2000). Por ejemplo, la exposición a la violencia en edades tempranas se asocia posteriormente con conductas antisociales (Wilson, Stover \& Berkowitz, 2009), como, también, ser víctima de maltrato a temprana edad (Braga, Gonçalves, Basto-Pereira \& Maia, 2017; Egeland, Yates, Appleyard \& van Dulmen, 2002).

Otro de los factores de riesgo relacionados con conductas antisociales, especialmente en la adolescencia, es la influencia de pares que presentan conductas antisociales (Dishion \& Patterson, 2006; Mahoney, Stattin \& Lord, 2004; Monahan, Steinberg \& Cauffman, 2009; Plazas et al., 2010; Vitaro, Brendgen \& Tremblay, 2000). Por ejemplo, un estudio con 362 adolescentes estadounidenses evidenció que la relación (entendida como amistad y afiliación) con pares antisociales tenía un efecto moderador entre una parentalidad negativa y el despliegue de conductas antisociales: si la relación con los pares es de carácter antisocial, el efecto de la crianza negativa aumentaba (Lansford, Criss, Pettit, Dodge \& Bates, 2003).

De esta forma, y teniendo en cuenta el efecto de la familia sobre la conducta de los sujetos en los primeros años de vida, el efecto de los pares con conductas antisociales en edades posteriores resulta un factor de riesgo fundamental para entender trayectorias y compromiso con conductas antisociales (Caicedo \& Jones, 2014; Ingoldsby et al., 2006; Vitaro et al., 2000). Un mecanismo de influencia de los pares sobre la aparición y mantención de la conducta antisocial es por medio de las normas de grupo. Se ha estudiado que, 
dentro de los grupos de pares, los niveles de tolerancia y aceptación hacia las conductas de violencia o agresión pueden explicar su desarrollo y mantención (Benbenishty, Astor, Zeira \& Vinokur, 2002; Caicedo \& Jones, 2014; Gaias, Lindstrom, White, Pettigrew \& Dumka, 2019). Considerando esto, Vitaro et al. (2015) observaron, en una muestra de 266 pares de gemelos de Montreal, Canadá, la existencia de factores genéticos comunes que influirían en la conducta antisocial y, a su vez, que podría ser moderada por las normas de grupo, evidenciando la importancia de los pares en estas conductas.

Si bien la influencia del grupo de pares sobre la generación de conductas antisociales en la adolescencia está reconocida en la literatura, pocos estudios han examinado el rol de la escuela como factor protector ante esta relación. Investigaciones en Estados Unidos han logrado identificar cómo la presencia de factores protectores reduce las probabilidades de manifestar conductas antisociales y de violencia en la adolescencia en población más vulnerable y con mayores niveles de riesgo (Kim et al., 2016; Stoddard, Zimmerman \& Bauermeister, 2012). Sin embargo, existe menos información sobre dicha relación en contextos sudamericanos y especialmente en Chile.

\section{La Escuela como Factor Protector de Conductas Antisociales y de Violencia}

Junto a la presencia de factores de riesgo que influyen en el desarrollo de conductas de riesgo, existen factores protectores que juegan un rol importante en su prevención. Como factores protectores se entienden aquellas variables de origen interno o externo que actúan como moderadores del impacto de los factores de riesgo en la conducta humana (Fergus \& Zimmerman, 2005). Dentro de las influencias internas, están las llamadas constitucionales, como el temperamento, las competencias emocionales y cognitivas (Sullivan \& Hirschfield, 2011), mientras que la familia, el barrio y la escuela son influencias de tipo externo, y pueden ser espacios de intervención para la prevención de conductas de riesgo (Catalano \& Hawkins, 1996). Para esto, es necesario crear en estos contextos instancias de participación e interacción en las que niños y jóvenes tengan la oportunidad de involucrarse activamente, lo que, a su vez, debe ser reconocido y reforzado de manera positiva por su entorno para que se produzca la adquisición de conductas prosociales que moderen los factores de riesgo (Catalano \& Hawkins, 1996; Torres \& Varela, 2013).

Es así como el espacio escolar, considerando la gran cantidad de tiempo que pasan allí los individuos durante las primeras etapas de sus vidas y, por ende, las múltiples influencias que reciben de dicho contexto, pueden jugar un rol fundamental en el desarrollo humano (Eccles \& Roeser, 2011). Para comprender mejor la influencia del espacio escolar sobre el desarrollo psicosocial de los estudiantes, es importante considerar, también, que los objetivos de la escuela como institución formadora ya no se reducen solo a la entrega de información por medio de procesos unidireccionales de interacción, sino que incluyen la responsabilidad de los actores educativos de promover procesos de desarrollo integral en los estudiantes (Casassus, 2002). De esta forma, se reconoce que aquellos contextos escolares que son capaces de satisfacer las necesidades sociales y emocionales de sus estudiantes permiten una mayor probabilidad de aprendizaje de los contenidos curriculares, junto a la generación de una mayor motivación por aprender y un aumento del compromiso con la escuela, reduciendo, de esta manera, deserciones, repitencias y expulsiones en la trayectoria escolar (Bruns et al., 2016; Catalano, Haggerty, Oesterle, Fleming \& Hawkins, 2004; Gaias et al., 2019; Hawkins, Catalano, Kosterman, Abbott \& Hill, 1999; Jimmerson, Nickerson, Mayer \& Furlong, 2012; Johnson, Crosnoe \& Elder Jr., 2001; Malecki \& Elliot, 2002).

\section{Oportunidades de Participar en la Escuela}

Participar e involucrarse en la vida escolar es un aspecto clave en la promoción del bienestar de los estudiantes. Para algunos autores (Catalano \& Hawkins, 1996), dicha participación con la escuela se entiende como un constructo que consta de dos principales componentes: (a) el apego, es decir, la mantención de relaciones afectivas cercanas y positivas con la comunidad escolar y (b) el compromiso escolar. Distintas investigaciones muestran que el apego escolar promueve un desarrollo psicosocial saludable y juega un importante rol en la socialización de conductas prosociales que pueden inhibir los problemas de conducta en niños y adolescentes (Catalano et al., 2004; da Silva, Lucato \& Rezende, 2016). Por medio de un análisis multinivel, Rovis, Jonkman y Basic (2016) encontraron, en una muestra de 1.927 adolescentes croatas, que manteniendo constantes las variables edad, género, antecedentes familiares y nivel individual de apego y compromiso escolar, aquellos estudiantes que acudían a escuelas donde se mostraban mayores niveles de apego escolar reportaban menores conductas de riesgo, tanto si provenían de contextos familiares adversos o no. 
Algunas investigaciones muestran que los estudiantes que sienten apego por su establecimiento escolar presentan mayor motivación por sus estudios y están en menor riesgo de exhibir problemas de conducta o presentar conductas delictuales (da Silva et al., 2016). Al contrario, un bajo apego escolar, en combinación con otros factores personales, sociales y contextuales, incrementan la probabilidad de comportamientos problemáticos en adolescentes. En base a esto, da Silva et al. (2016) estudiaron los niveles de apego escolar en 60 adolescentes, divididos en cuatro grupos: (a) estudiantes sin problemas de conducta, (b) estudiantes con problemas de conducta, (c) desertores escolares con problemas de conducta y (d) desertores sin problemas de conducta. Aquellos estudiantes que presentaban problemas de conducta, pero que todavía no habían desertado del colegio (b), tenían mayores niveles de apego escolar, en comparación con los desertores (c), lo cual podría mostrar como el apego escolar puede operar como factor protector de la deserción escolar en adolescentes con problemas conductuales. Asimismo, los jóvenes sin problemas de conducta que desertaron del sistema escolar (d) reportaron cuatro veces mayor apego a los profesores que aquellos desertores que sí presentaban problemas de conducta (c). De aquí se desprende que el apego escolar es un factor importante de considerar al prevenir posibles conductas antisociales.

Por su parte, Chapman, Buckley, Sheehan y Shochet (2013) examinaron siete programas de intervención escolar para la prevención de conductas de riesgo en estudiantes, cinco en Estados Unidos, uno en Australia y uno en Alemania. Luego de la revisión de las evaluaciones de estos programas, encontraron que aquellos que apuntaban a aumentar la conexión con la escuela para prevenir la toma de riesgos resultaron positivamente evaluados, demostrando cambios positivos tanto en apego escolar como en la disminución de conductas de riesgo, y también en ambos.

En cuanto a información que se dispone sobre estudiantes chilenos, un estudio encontró como predictores del bienestar emocional de los estudiantes dos componentes importantes: a) la percepción de apoyo e inclusión por parte de compañeros, y b) profesores disponibles y dispuestos a dialogar, que manifiesten preocupación por las dificultades académicas y personales de los estudiantes (Berger et al., 2009), ambos componentes importantes en el constructo de apego escolar (Chapman et al., 2013).

El apego escolar resulta, entonces, fundamental para prevenir diversas conductas de riesgo en los niños y adolescentes, así como para evitar conductas antisociales y promover un clima escolar positivo que favorezca a los estudiantes en distintos ámbitos de su desarrollo. En el contexto chileno se han promovido diferentes políticas y legislaciones que buscan fortalecer estas dimensiones, tales como la ley de inclusión escolar (Chile, Ministerio de Educación, 2015), la ley sobre violencia escolar (Chile, Ministerio de Educación, 2011) y otros indicadores de calidad, como el clima de convivencia escolar (Agencia de Calidad de la Educación, 2016; Ley $\mathrm{N}^{\circ} 20.536,2011$; Ley $\left.\mathrm{N}^{\circ} 20.845,2015\right)$. No obstante, falta todavía evidenciar cómo podrían operar estas dimensiones sobre conductas antisociales en adolescentes.

Teniendo en cuenta el valor del espacio escolar como promotor del desarrollo, la presente investigación buscó profundizar en los factores protectores, desde la percepción individual, de elementos escolares que puedan moderar el efecto de factores de riesgo, como es la interacción con pares antisociales, sobre conductas de carácter antisocial en adolescentes.

La hipótesis principal fue que mantener vínculos sólidos con los espacios donde se desenvuelve el niño o adolescente, como la escuela, la familia o la comunidad, opera como factor protector sobre conductas de riesgo (Catalano et al., 2004; Catalano \& Hawkins, 1996; Hawkins, 1999). De esta manera, mantenerse activamente involucrado en estos espacios, a través de un vínculo emocional positivo, prevendría que los individuos ejerzan conductas que transgreden las normas sociales aceptadas (Hawkins, 1999). En particular, este estudio buscó analizar el efecto de una percepción de mayor apego con la escuela como moderador del efecto de pares sobre las conductas antisociales en adolescentes chilenos.

\section{Método}

\section{Participantes}

La muestra fue estratificada con afijación igual (Canales, 2006), al asignar un mismo número de elementos por estrato. La población del estudio se estratificó por nivel socioeconómico (NSE), según el Sistema de Medición de la Calidad de la Educación (SIMCE) de año 2010. En particular, se utilizó la variable grupo socioeconómico al que pertenece el colegio, se revisó la correspondencia con el NSE y, de esta población, se realizó selección aleatoria de 3 colegios por estrato. Así, la muestra estuvo compuesta por 815 
escolares de $7^{\circ}$ año básico a IV año medio, correspondiente a los ciclos de enseñanza básica y secundaria del país, $46,6 \%$ mujeres, con una edad promedio de 15,11 años $(D E=1,78)$, provenientes de 15 establecimientos escolares de cinco diferentes NSE de la Región Metropolitana.

\section{Instrumento}

Se usó la adaptación en Chile de Communities That Care Youth Survey (Arthur, Hawkins, Pollard, Catalano, \& Baglioni, 2002; Torres-Vallejos \& Sosa, 2013) para medir las siguientes escalas:

Conducta Antisocial en Jóvenes (CA). Esta es la variable dependiente del estudio. Esta escala está conformada por 10 ítems que miden el autorreporte de conductas antisociales, tales como agredir a alguien, robar, dañar propiedad ajena o ir bajo efectos de drogas y alcohol al colegio. Se encuentra medida en una escala tipo Likert que va desde 0 (nunca) a $7(40$ o + veces). Ejemplos de ítems son: ¿Cuántas veces durante el año has ido a la escuela borracho/a o drogado/a?; ¿Cuántas veces durante el año has dañado o destruido a propósito propiedad que no es tuya (sin contar propiedad familiar)?; ¿Cuántas veces durante el año te has llevado algo de una tienda sin pagarlo?. Para este estudio, la escala presenta un adecuado indicador de consistencia interna ( $\alpha$ de Cronbach $=0,84$ ).

Influencia de pares con conductas antisociales. Para las variables independientes del estudio, se consideraron dos escalas por separado para valorar la interacción con pares que presentan conductas de carácter antisocial:

Relación con Pares Antisociales (PA). Esta escala está compuesta por seis ítems que evalúen el autorreporte en relación con diferentes conductas antisociales de amigos/as cercanos/as, considerada como un proxy de influencia de pares. Es medida a través de una escala Likert de 5 puntos, donde 0 es Ningún amigo y 4, 4 de mis amigos. Las conductas consideradas son: suspensión del colegio, porte de armas al interior del colegio, venta de drogas, robo de autos, haber sido arrestado o haber desertado del colegio. Ejemplos de ítems son: Piensa en tus cuatro mejores amigos/as (aquellos a los/as que te sientes más cercano/a). Durante este año, ¿cuántos de tus mejores amigos/as han sido suspendidos?; ¿Cuántos de tus mejores amigos/as han vendido drogas ilegales?; ¿Cuántos de tus mejores amigos/as han sido arrestados/as?. Para este estudio, la escala presenta un adecuado indicador de consistencia interna (a de Cronbach $=0,82$ ).

Consumo Drogas Pares (CD). Esta escala está compuesta por cuatro ítems que miden el autorreporte de relación con amigos cercanos que consumen drogas legales e ilegales, también como un proxy de influencia de pares. Esta medida usa una escala Likert de 5 opciones de respuesta, donde 0 es Ninguno de mis amigos y 4 es 4 de mis amigos. Ejemplos de los ítems son: Piensa en tus cuatro mejores amigos/as (aquellos a los/as que te sientes más cercano/a). Durante este año, ¿cuántos de tus mejores amigos/as han probado cerveza, vino o licores fuertes (por ejemplo, pisco, ron o vodka) sin que sus padres se dieran cuenta?; "Durante este año, ¿cuántos de tus mejores amigos/as han consumido pasta base, cocaína, anfetaminas u otras drogas ilegales?. Un puntaje más alto indica mayor reporte de amigos cercanos que reportan consumo. Para este estudio, la escala presenta un adecuado indicador de consistencia interna ( $\alpha$ de Cronbach $=0,77)$.

Oportunidades y Recompensas de Participación en la Escuela (ORPE). Esta escala consta de nueve ítems que miden el grado de involucramiento, forma y opciones de ser parte del establecimiento educacional. Si bien la escala original consta de dos subescalas, para efectos del estudio se fusionaron ambos aspectos de la escala como un solo factor protector, siguiendo lo realizado por otros autores (Feinberg, Ridenour \& Greenberg, 2007). Las preguntas fueron respondidas a través de una escala Likert de 4 puntos, desde $0(N o)$ hasta $3(S \hat{\imath})$, es decir, a mayor valor hay mayor involucramiento con el colegio. Ejemplos de ítems son: En mi establecimiento educacional los profesores me felicitan cuando me esfuerzo y trabajo bien; En mi establecimiento educacional se ofrece a los estudiantes muchas oportunidades de hablar con los profesores de forma individual; En mi establecimiento educacional los estudiantes tienen muchas oportunidades de ayudar a decidir cosas, como actividades de clase y normas de convivencia. Para este estudio, la escala presenta un apropiado coeficiente de consistencia interna ( $\alpha$ de Cronbach $=0,75$ ).

Finalmente, como variables de control se incluyeron el sexo como variable dicotómica $(0=$ hombre; $1=$ mujer) y la edad como variable continua. 


\section{Procedimiento}

La participación del estudio fue de carácter voluntario, por medio de un consentimiento informado firmado por los adolescentes, consentimiento pasivo de sus padres y apoderados (a través de un comunicado donde se podían negar a participar si así lo deseaban) y consentimiento informado de los directores y centros de padres. El estudio fue aprobado por el Comité de Ética de la Universidad de Santiago de Chile, institución donde se originó este estudio.

La aplicación del instrumento se hizo de forma masiva en cada curso, con presencia de dos examinadores del equipo de investigación y un representante del colegio, la cual duraba una hora en promedio.

\section{Plan de Análisis}

En una primera etapa se llevaron a cabo análisis descriptivos para explorar las variables y su relación por medio de correlación de Pearson. Posteriormente, se llevó a cabo un análisis de regresión múltiple, siguiendo las orientaciones de los modelos de resiliencia con el método Enter. Estos modelos de análisis están basados en salud pública para identificar factores protectores en función de factores y conductas de riesgo (Fergus \& Zimmerman, 2005). El orden de los predictores fue: (a) variables de control (sexo y edad), (b) influencia de pares con conductas antisociales (factor de riesgo), (c) Oportunidades y recompensas de participación en la escuela (factor protector) y (d) la interacción entre ambos. Los predictores fueron centrados en su media para evitar multicolinealidad. Respecto de los valores perdidos, la prueba Little's MCAR, $\left.\chi^{2}(1361, N=815)=1805,43, p=0,001\right)$ no logra evidenciar que los valores estén completamente perdidos de forma aleatoria; sin embargo, estos representan menos del $2 \%$.

\section{Resultados}

El promedio de edad de los participantes fue de 15,11 años $(D E=1,78)$. Como se observa en la Tabla 1 , todas las escalas presentaron rangos de respuesta distintos: la escala de CA presenta una media más baja que las demás variables, y considerada, de acuerdo con su escala, como una baja prevalencia de participación en acciones de este tipo. La PA tiene una media también baja para esta escala. A su vez, el CD promedio es bajo en base a la escala usada.

Tabla 1

Estadísticos Descriptivos

\begin{tabular}{lrrrrr}
\hline \multicolumn{1}{c}{ Variable } & \multicolumn{1}{c}{$M$} & \multicolumn{1}{c}{$D$} & $\%$ & Mínimo & Máximo \\
\hline Sexo (mujer) & \multicolumn{1}{c}{ - } & \multicolumn{1}{c}{-} & 58,8 & 1 & 2 \\
Edad & 15,11 & 1,78 & - & 12 & 20 \\
Conducta antisocial personal en jóvenes (CA) & 0,25 & 0,60 & - & 0 & 7 \\
Relación con pares antisociales (PA) & 0,42 & 0,59 & - & 0 & 4 \\
Consumo drogas pares (CD) & 1,19 & 1,08 & - & 0 & 4 \\
Oportunidades y recompensas de & 1,93 & 0,48 & - & 0 & 3 \\
participación en la escuela (ORPE) & & & & & \\
\hline
\end{tabular}

En la Tabla 2 se presentan las correlaciones de Pearson entre las escalas del estudio y las variables de control. Como se puede observar, existe una correlación positiva entre las CA, PA y CD y, a su vez, una correlación negativa con ORPE. Esta última variable también se correlaciona de forma negativa con PA y CD. Esto indica que, efectivamente, a mayor percepción de apego con la escuela existe un menor reporte de conductas antisociales y relación con pares que también las realizan, como también con aquellos que consumen drogas. 
Tabla 2

Correlaciones de Pearson entre los Factores

\begin{tabular}{lcccccc}
\hline \multicolumn{1}{c}{ Variable } & Edad & Sexo & CA & PA & CD & ORPE \\
\hline Edad & - & & & & & \\
Sexo & $0,14^{* *}$ & - & & & & \\
Conducta antisocial en la escuela (CA) & $-0,02$ & $-0,13^{* *}$ & - & & & \\
Relación con pares antisociales (PA) & $-0,01$ & $-0,14^{* *}$ & $0,47^{* *}$ & - & & \\
Consumo drogas pares (CD) & $-0,26^{* *}$ & 0,05 & $0,29^{* *}$ & $0,57^{* *}$ & - & \\
Oportunidades y recompensas de & $-0,08^{* *}$ & $0,11^{* *}$ & $-0,21^{* *}$ & $-0,17^{* *}$ & $-0,23^{* *}$ & - \\
participación con la escuela (ORPE) & & & & & &
\end{tabular}

Nota. ${ }^{* *} p<0,01 ;{ }^{*} p<0,05 . N$ es 808 a 812 , según la correlación.

Tabla 3

Regresión Múltiple de Pares Antisociales y Oportunidades y Recompensas de Participación en la Escuela en Conductas Antisociales

\begin{tabular}{|c|c|c|c|c|c|c|c|c|c|c|c|c|}
\hline & \multicolumn{3}{|c|}{ Modelo 1} & \multicolumn{3}{|c|}{ Modelo 2} & \multicolumn{3}{|c|}{ Modelo 3} & \multicolumn{3}{|c|}{ Modelo 4} \\
\hline & B & $E E$ & $B$ & B & $\mathrm{EE}$ & $B$ & B & $\mathrm{EE}$ & B & B & $E E$ & $B$ \\
\hline Sexo (mujer) & $-0,15$ & 0,04 & $-0,13^{* *}$ & $-0,09$ & 0,04 & $-0,07^{*}$ & $-0,07$ & 0,04 & $-0,06$ & $-0,09$ & 0,04 & $-0,07^{*}$ \\
\hline Edad & 0,00 & 0,01 & 0,00 & $-0,00$ & 0,01 & $-0,01$ & $-0,00$ & 0,01 & $-0,02$ & $-0,01$ & 0,01 & $-0,03$ \\
\hline $\mathrm{PA}$ & & & & 0,43 & 0,04 & $0,43^{* *}$ & 0,43 & 0,04 & $0,42^{* *}$ & 0,36 & 0,04 & $0,36^{*}$ \\
\hline $\mathrm{CD}$ & & & & 0,03 & 0,02 & 0,05 & 0,01 & 0,02 & 0,03 & 0,03 & 0,02 & 0,06 \\
\hline ORPE & & & & & & & $-0,16$ & 0,04 & $-0,13^{* *}$ & $-0,17$ & 0,06 & $-0,14^{* *}$ \\
\hline Sexo*ORPE & & & & & & & & & & 0,08 & 0,08 & 0,04 \\
\hline Edad*ORPE & & & & & & & & & & 0,01 & 0,02 & 0,02 \\
\hline $\mathrm{PA}^{*} \mathrm{ORPE}$ & & & & & & & & & & $-0,28$ & 0,06 & $-0,19^{* *}$ \\
\hline $\mathrm{CD}^{*} \mathrm{ORPE}$ & & & & & & & & & & 0,02 & 0,05 & 0,02 \\
\hline Constante & 0,31 & 0,18 & & 0,35 & 0,17 & & 0,37 & 0,17 & & 0,44 & 0,17 & \\
\hline$R^{2}$ ajustado & & & 0,01 & & & 0,22 & & & 0,24 & & & 0,26 \\
\hline Cambio en $R^{2}$ & & & 0,02 & & & 0,21 & & & 0,02 & & & 0,03 \\
\hline$f^{2}$ & & & 0,11 & & & 0,28 & & & 0,32 & & & 0,35 \\
\hline$F$ & & & 6,70 & & & 107,57 & & & 15,94 & & & 8,96 \\
\hline$G l$ & & & 803 & & & 801 & & & 800 & & & 796 \\
\hline
\end{tabular}

Nota. PA: Pares antisociales; CD: Consumo drogas pares; ORPE: Oportunidades y recompensas de participación en la escuela.

${ }^{* *} p<0,01 ;{ }^{*} p<0,05$

Los resultados del análisis jerárquico con el modelo nulo evidencian un coeficiente de correlación intraclase de 1,26\% de variabilidad entre colegios. En base a esto, y considerando el bajo tamaño muestral de nivel escuela para estimar el error estándar, se llevaron a cabo análisis de regresión múltiple. En los análisis de regresión múltiple se aplicaron los modelos de resiliencia. Como se observa en la Tabla 3, el 
primer modelo muestra que tanto las variables sexo y edad resultan ser significativas en relación con las conductas antisociales $\left(R^{2}=0,01\right)$, explicando de mejor forma estas variables cuando se considera en el segundo modelo los dos factores de riesgo $\left(R^{2}=0,22\right)$. De la misma forma, el tamaño del efecto del modelo 2 es mucho mayor que el del primero $\left(f^{2}=0,11 ; f^{2}=0,28\right.$, respectivamente). Al introducir el factor protector en el modelo 4, el modelo mejora $\left(R^{2}=0,24\right)$, al igual que en el modelo 5, el cual suma todos los factores de interacción $\left(R^{2}=0,26\right)$. Finalmente, el modelo 4 tiene un tamaño del efecto mediano y el modelo 5 un efecto grande (Cárdenas Castro \& Arancibia Martini, 2014).

El factor de interacción se puede apreciar en la Figura 1. Existe una asociación directa entre la relación con PA y el reporte de CA. Sin embargo, esta relación varía según los niveles de percepción de ORPE, la cual específicamente se ve moderada en aquellos estudiantes que reportan una mayor percepción de apego escolar.

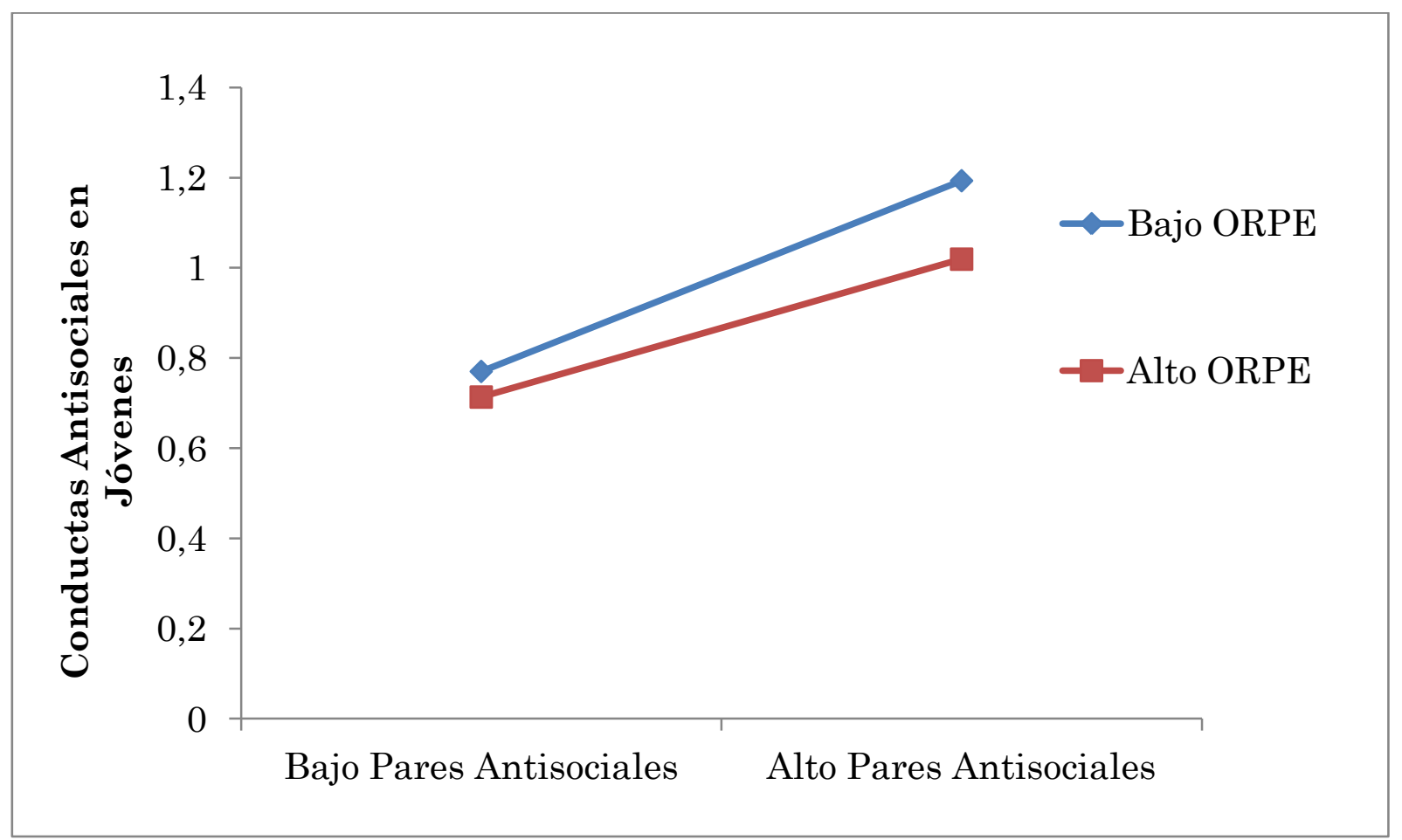

Figura 1. Conducta antisocial explicada por la interacción entre pares antisociales y la percepción de oportunidades y recompensas de particicpación en la escuela (ORPE).

\section{Discusión}

Los resultados del estudio confirman la importancia de la percepción de un mayor apego con la escuela, como un aspecto clave para el desarrollo de CA en adolescentes. En particular, la percepción de una escuela en donde los adolescentes se sientan más involucrados juega un rol fundamental en su desarrollo, no solo por la cantidad de tiempo que pasan en ella, sino también porque constituye un espacio de protección y socialización (Eccles \& Roeser, 2011), donde construyen gran parte de su identidad. En este espacio conviven múltiples ámbitos de la vida, tales como el académico y las relaciones con pares y otros adultos significativos, los cuales ejercen diferentes formas de influencia en la vida de los estudiantes.

Es en la etapa escolar, y sobre todo en la adolescencia, que las relaciones entre pares juegan un rol fundamental en el desarrollo socioemocional (Plazas et al., 2010; Rojas Andrade \& Leiva Bahamondes, 2015), pudiendo operar como factores de riesgo o bien como factores protectores (Fergus \& Zimmerman, 2005). Bajo el modelo de Fergus y Zimmerman (2005), estos factores son dinámicos, pudiendo actuar como factores de riesgo o de protección, dependiendo de los efectos que tengan sobre el individuo y su desarrollo (Catalano \& Hawkins, 1996; Torres \& Varela, 2013). Aun así, existen algunas variables consensuadas por 
la evidencia empírica, tales como la violencia como factor de riesgo para el consumo de drogas e involucramiento en CA y el apego con la escuela y conducta prosocial como factores protectores (Kim et al., 2016).

Dentro del modelo de desarrollo social en el cual se basan las variables del estudio, el CD de pares y personal está relacionado con múltiples factores, por lo que la influencia de pares no es lineal (Catalano \& Hawkins, 1996). Según esta teoría, estas conductas aparecen en la infancia y adolescencia, pero su mantención se explica por la disponibilidad de sustancias en el medio cercano y creencias favorables hacia ellas, no solo del individuo, sino también de sus pares, quienes también influyen en la selección de amistades (Henneberger, Mushonga \& Preston, 2020). Estudios más recientes indican que también se asocian al autocontrol y la toma de riesgos (Lloyd \& Döring, 2019). Por su parte, en el caso de la influencia de pares, estudios como los de Vitaro et al. (2015) muestran cómo los otros significativos pueden moderar la $\mathrm{CA}$, relacionada con una actitud favorable hacia ella, incluso cuando existen factores genéticos o de socialización primaria. Estas son variables importantes de considerar en futuros estudios, que también podrían explicar el consumo de drogas en el modelo explicativo.

Por otro lado, los resultados del estudio apoyan la evidencia existente acerca de la importancia de promover la conexión con la escuela para reducir el impacto de ciertos factores de riesgo sobre conductas de riesgo en adolescentes (Chapman et al., 2013). De aquí se desprende información importante de considerar al momento de diseñar programas de intervención que favorezcan los procesos de desarrollo integral de niños, niñas y adolescentes. Según esto, los programas que promueven el involucramiento de los estudiantes hacia su comunidad escolar tienen mayores probabilidades de ser exitosos a la hora de prevenir conductas de riesgo en adolescentes. Según la revisión de Chapman et al. (2013), las estrategias usadas en las intervenciones efectivas contemplan extensos cambios en el sistema escolar en su conjunto, así como formación a los docentes para que estos implementen cambios en sus prácticas en el aula con el fin de generar un buen clima de aula, provisto de espacios para la interacción positiva entre los miembros de la comunidad. Futuras investigaciones podrían contribuir a especificar qué tipo de prácticas son más efectivas y esclarecer de qué manera se pueden lograr estos cambios a nivel de sistema escolar, considerando las particularidades y necesidades específicas de cada uno.

Por otra parte, es posible que en el espacio escolar coexistan múltiples y diversas influencias, tanto las que promueven las relaciones interpersonales positivas y un buen clima escolar, como también aquellas que pueden producir formas de interacción negativas, que no promueven el bienestar de los estudiantes. Por lo mismo, este fenómeno debe ser comprendido más allá de los aspectos individuales de los actores involucrados, desde una mirada interpersonal y grupal (Potocnjak, Berger \& Tomicic, 2011). Esta mirada más ecológica permite, entonces, relevar aspectos clave de la vida escolar para prevenir estas conductas, tales como el apego escolar (Catalano et al., 2004; Catalano \& Hawkins, 1996; Hawkins, 1999), en el marco de fortalecer un mejor clima escolar en la escuela. En este sentido, programas de prevención en el contexto escolar podrían desarrollar metodologías que incentiven esta dimensión en la escuela y evidenciar cómo la escuela puede transformarse en un factor protector, según se ha visto en otros contextos (Chapman et al., 2013). De hecho, las intervenciones y tratamientos que han resultado para prevenir las conductas antisociales a largo plazo han mostrado tener un efecto, en especial cuando son ejecutadas por investigadores, aunque todavía con un efecto moderado (Sawyer, Borduin \& Dopp, 2015). Esto incentiva la posibilidad de explorar otras dimensiones de la vida en la escuela que puedan contribuir a ello.

De la misma forma, las políticas nacionales de prevención podrían fortalecer el clima escolar y la participación estudiantil, la cual puede operar como un factor protector, consistente con otros estudios (Catalano et al., 2004; da Silva et al., 2016; Rovis et al., 2016). Para algunos autores el apego escolar puede ser considerado una dimensión del clima escolar, reconociendo la complejidad y multidimensionalidad de este constructo (Thapa, Cohen, Guffey \& Higgins-D'Allessandro, 2013; Wang \& Degol, 2016) y su impacto en problemas de conducta (Reaves, McMahon, Duffy \& Ruiz, 2018). En base a los resultados del estudio se puede observar un efecto del apego escolar como factor protector de la influencia de los pares. Sin embargo, su efecto, si bien es significativo, todavía sigue siendo bajo. Según esto, se puede hipotetizar que es necesario explorar sobre otras dimensiones del clima escolar para incrementar su efecto sobre los estudiantes. A pesar de esto, la experiencia escolar tiene un significativo efecto en el desarrollo humano (Eccles \& Roeser, 2011; Roeser et al., 2000) y, al mismo tiempo, permite generar vínculos con instituciones formales de la sociedad. Tal como ha descrito Hirschi (1998), cuando los sujetos establecen este tipo de conexiones con instituciones como la escuela aumenta la probabilidad de incorporar normas de comportamiento que impacten en CA, lo cual podría explicar el rol de la escuela como moderador. 
A pesar de estos resultados, hay algunas limitaciones que mencionar. Por una parte, solo se usaron datos de autorreporte como fuente de información por parte de los estudiantes. Si bien otras fuentes podrían complementar la información recolectada, estos datos resultan valiosos para conocer la percepción de los alumnos. De la misma forma, los datos son de carácter transversal, limitando la capacidad de incorporar diferencias en el tiempo. Futuros estudios podrían examinar variaciones a lo largo del tiempo para comprobar si se mantienen los mecanismos identificados con este estudio. El sexo y la edad de las participantes fueron incluidos como variables de control. Sin embargo, el NSE no fue incorporado en el estudio, debido a que la información que se tenía correspondía solo a los colegios de la muestra y no a nivel de cada participante. Futuras investigaciones podrían incorporar este dato a nivel individual para examinar su grado de asociación con las CA. Otra limitación del estudio fue que, si bien se llevaron a cabo varios procedimientos de resguardo ético con los adolescentes, incorporando a directivos, centros de padres y estudiantes, el consentimiento de los apoderados fue de carácter pasivo. Sería recomendable fortalecer un rol más activo de los mismos para próximas evaluaciones, entendiendo la importancia de su autorización en investigaciones con menores de edad. Finalmente, los datos provienen de un contexto urbano de Chile, lo cual no necesariamente representa la diversidad cultural y étnica del país. Contar con una muestra más grande y diversa de colegios en futuros estudios permitiría agregar otras hipótesis de mayor complejidad, como por ejemplo, tipos de colegios -urbano versus rural- y desarrollar análisis jerárquicos. A pesar de estas limitaciones, el presente estudio logra mostrar el efecto de una mayor percepción de apego con la escuela como un factor protector de CA, replicando los resultados encontrados en otras partes del mundo, lo cual también refuerza la importancia de la vida en la escuela para el desarrollo de niños, niñas y adolescentes.

\section{Referencias}

Arthur, M. W., Hawkins, J. D., Pollard, J. A., Catalano, R. F. \& Baglioni Jr., A. J. (2002). Measuring risk and protective factors for substance use, delinquency, and other adolescent problem behaviors: The Communities That Care Youth Survey. Evaluation Review, 26, 575-601. https://doi.org/10.1177/0193841X0202600601

Benbenishty, R., Astor, R. A., Zeira, A. \& Vinokur, A. D. (2002). Perceptions of violence and fear of school attendance among junior high school students in Israel. Social Work Research, 26, 71-87. https://doi.org/10.1093/swr/26.2.71

Berger, C., Milicic, N., Alcalay, L., Torretti, A., Arab, M. P. \& Justiniano, B. (2009). Bienestar socio-emocional en contextos escolares: la percepción de estudiantes chilenos. Estudios sobre Educación, 17, 21-43. Recuperado de https://dadun.unav.edu/bitstream/10171/9839/3/17Eb.pdf

Braga, T., Gonçalves, L. C., Basto-Pereira, M. \& Maia, Â. (2017). Unraveling the link between maltreatment and juvenile antisocial behavior: A meta-analysis of prospective longitudinal studies. Aggression and Violent Behavior, 33, 37-50. https://doi.org/10.1016/j.avb.2017.01.006

Bronfenbrenner, U. (1994). Ecological models of human development. En T. Husén \& T. N. Postlethwaite (Eds.), The international encyclopedia of education ( $2^{\text {a }}$ ed., pp. 1643-1647). Oxford, Reino Unido: Pergamon Press.

Bruns, E. J., Duong, M. T., Lyon, A. R., Pullmann, M. D., Cook, C. R., Cheney, D. \& McCauley, E. (2016). Fostering SMART partnerships to develop an effective continuum of behavioral health services and supports in schools. American Journal of Orthopsychiatry, 86, 156-170. https://doi.org/10.1037/ort0000083

Caicedo, B. \& Jones, K. (2014). The role of the neighborhood, family and peers regarding Colombian adolescents' social context and aggressive behavior. Revista de Salud Pública, 16, 208-220. https://doi.org/10.15446/rsap.v16n2.38983

Canales, M. (2006). Metodologías de la investigación social. Santiago, Chile: LOM.

Cárdenas Castro, M. \& Arancibia Martini, H. (2014). Potencia estadística y cálculo del tamaño del efecto en G*Power: complementos a las pruebas de significación estadística y su aplicación en psicología. Salud \& Sociedad, 5, 210-224. https://doi.org/10.22199/S07187475.2014.0002.00006

Casassus, J. (2002). Cambios paradigmáticos en educación. Revista Brasileira de Educação, 20, 48-59. https://doi.org/10.1590/S141324782002000200004

Catalano, R. F., Haggerty, K. P., Oesterle, S., Fleming, C. B. \& Hawkins, J. D. (2004). The importance of bonding to school for healthy development: findings from the Social Development Research Group. Journal of School Health, 74, 252-261. https://doi.org/10.1111/j.1746-1561.2004.tb08281.x

Catalano, R. F. \& Hawkins, J. D. (1996). The social development model: A theory of antisocial behavior. En J. D. Hawkins (Ed.), Delinquency and crime: Current theories (pp. 149-197). Cambridge, Reino Unido: Cambridge University Press.

Chapman, R. L., Buckley, L., Sheehan, M. \& Shochet, I. (2013). School-based programs for increasing connectedness and reducing risk behavior: A systematic review. Educational Psychology Review, 25, 95-114. https://doi.org/10.1007/s10648-013-9216-4

Chile, Ministerio de Educación, Agencia de Calidad de la Educación. (2016). Desarrollo Personal y Social: Otros indicadores de calidad educativa. Recuperado de https://bibliotecadigital.mineduc.cl/bitstream/handle/20.500.12365/4577/Desarrollopersonalsocial.pdf?sequence=1\&isAllowed=y

Chile, Ministerio de Educación (2011, Septiembre 17). Ley $N^{\circ}$ 20.536. Sobre Violencia Escolar. Santiago, Chile: Diario Oficial de la República de Chile, Santiago, Chile, 17 de septiembre de 2011.

Chile, Ministerio de Educación (2015, Junio 8). Ley $N^{\circ} 20.845$ de Inclusión Escolar que regula la admisión de los y las estudiantes, elimina el financiamiento compartido y prohíbe el lucro en establecimientos educacionales que reciben fondos del estado. Santiago, Chile: Diario Oficial de la República de Chile.

Chile, Ministerio de Planificación (2009, Septiembre 12). Ley $N^{\circ}$ 20.379. Crea el sistema intersectorial de protección social e institucionaliza el subsistema de protección integral a la infancia "Chile Crece Contigo". Santiago, Chile: Diario Oficial de la República de Chile. 
da Silva, J., Lucato, A. \& Rezende, M. (2016). School bonding of adolescent offenders. Paidéia, 26(63), 91-100. https://doi.org/10.1590/1982-43272663201611

De la Peña Fernández, M. E. (2010). Conducta antisocial en adolescentes: factores de riesgo y de protección (Tesis de Doctorado, Universidad Complutense de Madrid, España). Recuperado de https://eprints.ucm.es/12024/1/T28264.pdf

Dishion, T. J. \& Patterson, G. R. (2006). The development and ecology of antisocial behavior in children and adolescents. En D. Cicchetti \& D. J. Cohen (Eds.), Developmental psychopathology. Volume 3: Risk, disorder, and adaptation (2a ed., pp. 503-541). Hoboken, NJ: John Wiley \& Sons. https://doi.org/ 10.1002/9780470939406.ch13

Dishion, T. J. \& Patterson, G. R. (2016). The development and ecology of antisocial behavior: Linking etiology, prevention, and treatment. En D. Cicchetti (Ed.), Developmental psychopathology, Vol. 3: Maladaptation and psychopathology (3a ed., pp. 647678). Hoboken, NJ: John Wiley \& Sons. https://doi.org/10.1002/9781119125556.devpsy315

dos Santos, W. S., Holanda, L. C., Meneses, G. O., Luengo, M. A. \& Gomez-Fraguela, J. A. (2019). Antisocial behaviour: A unidimensional or multidimensional construct? Avances en Psicología Latinoamericana, 37, 13-27. https://doi.org/10.12804/revistas.urosario.edu.co/apl/a.5105

Eccles, J. S. \& Roeser, R. W. (2011). Schools as developmental contexts during adolescence. Journal of Research on Adolescence, 21, 225-241. https://doi.org/10.1111/j.1532-7795.2010.00725.x

Egeland, B., Yates, T., Appleyard, K. \& van Dulmen, M. (2002). The long-term consequences of maltreatment in the early years: A developmental pathway model to antisocial behavior. Children's Services, 5, 249-260. https://doi.org/10.1207/s15326918cs0504_2

Eleni, D. \& Giotsa, A. (2018). Early detection of externalizing problems in preschool children according to their teachers. Psychology Research, 8, 60-73. https://doi.org/10.17265/2159-5542/2018.02.003

Espelage, D., Anderman, E. M., Brown, V. E., Jones, A., Lane, K. L., McMahon, S. D. ... Reynolds, C. R. (2013). Understanding and preventing violence directed against teachers: Recommendations for a national research, practice, and policy agenda. American Psychologist, 68, 75-87. https://doi.org/10.1037/a0031307

Farrington, D. P. (1995). The development of offending and antisocial behaviour from childhood: Key findings from the Cambridge Study in Delinquent Development. Journal of Child Psychology and Psychiatry, 36, 929-964. https://doi.org/10.1111/j.1469-7610.1995.tb01342.x

Farrington, D. P. (2005). Childhood origins of antisocial behavior. Clinical Psychology \& Psychotherapy, 12, 177-190. https://doi.org/10.1002/cpp.448

Feinberg, M. E., Ridenour, T. A. \& Greenberg, M. T. (2007). Aggregating indices of risk and protection for adolescent behavior problems: The Communities That Care Youth Survey. Journal of Adolescent Health, 40, 506-513. https://doi.org/10.1016/j.jadohealth.2006.09.002

Fergus, S. \& Zimmerman, M. A. (2005). Adolescent resilience: A framework for understanding healthy development in the face of risk. Annual Review of Public Health, 26, 399-419. https://doi.org/10.1146/annurev.publhealth.26.021304.144357

Gaias, L. M., Lindstrom Johnson, S., White, R. M. B., Pettigrew, J. \& Dumka, L. (2019). Positive school climate as a moderator of violence exposure for Colombian adolescents. American Journal of Community Psychology, 63, 17-31. https://doi.org/10.1002/ajcp.12300

Garaigordobil Landazabal, M. (2005). Conducta antisocial durante la adolescencia: correlatos socio-emocionales, predictores y diferencias de género. Psicología Conductual, 13, 197-215. Recuperado de https://www.behavioralpsycho.com/wpcontent/uploads/2020/04/02.Garaigordobil_13-2oa-1.pdf

Hawkins, J. D. (1999). Preventing crime and violence through Communities that Care. European Journal on Criminal Policy and Research, 7, 443-458. https://doi.org/10.1023/A:1008769321118

Hawkins, J. D., Catalano, R. F., Kosterman, R., Abbott, R. \& Hill, K. G. (1999). Preventing adolescent health-risk behaviors by strengthening protection during childhood. Archives of Pediatrics \& Adolescent Medicine, 153, 226-234. https://doi.org/10.1001/archpedi.153.3.226

Hawkins, J. D., Catalano, R. F. \& Miller, J. Y. (1992). Risk and protective factors for alcohol and other drug problems in adolescence and early adulthood: Implications for substance abuse prevention. Psychological Bulletin, 112, 64-105. https://doi.org/10.1037/0033-2909.112.1.64

Hein, A. \& Barrientos, G. (2004). Violencia y delincuencia juvenil: comportamientos de riesgo autorreportados y factores asociados. Santiago, Chile: Fundación Paz Ciudadana.

Henneberger, A. K., Mushonga, D. R. \& Preston, A. M. (2020). Peer influence and adolescent substance use: A systematic review of dynamic social network research. Adolescent Research Review. Anticipo en línea de la publicación. https://doi.org/10.1007/s40894019-00130-0 Recuperado de https://link.springer.com/article/10.1007/s40894-019-00130-0\#citeas

Hirschi, T. (1998). A control theory of delinquency. En F. P. Williams III \& M. D. McShane (Eds.), Criminology theory: Selected classic readings, 2nd edition (pp. 289-305). Cincinnati, OH: Anderson Publishing.

Ingoldsby, E. M., Shaw, D. S., Winslow, E., Schonberg, M., Gilliom, M. \& Criss, M. M. (2006). Neighborhood disadvantage, parentchild conflict, neighborhood peer relationships, and early antisocial behavior problem trajectories. Journal of Abnormal Child Psychology, 34, 293-309. https://doi.org/10.1007/s10802-006-9026-y

Jimmerson, S. R., Nickerson, A. B., Mayer, M. J. \& Furlong, M. J. (Eds.) (2012). The handbook of school violence and school safety. International research and practice $\left(2^{\mathrm{a}}\right.$ ed). New York, NY: Routledge.

Johnson, M. K., Crosnoe, R. \& Elder Jr., G. H. (2001). Students' attachment and academic engagement: The role of race and ethnicity. Sociology of Education, 74, 318-340. https://doi.org/10.2307/2673138

Kim, B. K. E., Gilman, A. B., Hill, K. G. \& Hawkins, J. D. (2016). Examining protective factors against violence among high-risk youth: Findings from the Seattle Social Development Project. Journal of Criminal Justice, 45, 19-25. https://doi.org/10.1016/j.jcrimjus.2016.02.015

Lansford, J. E., Criss, M. M., Pettit, G. S., Dodge, K. A. \& Bates, J. E. (2003). Friendship quality, peer group affiliation, and peer antisocial behavior as moderators of the link between negative parenting and adolescent externalizing behavior. Journal of Research on Adolescence, 13, 161-184. https://doi.org/10.1111/1532-7795.1302002

Lloyd, A. \& Döring, A. K. (2019). When do peers influence males' risk taking? Examining decision making under conditions of risk and ambiguity. Journal of Behavioral Decision Making, 32(5), 613-626. https://doi.org/10.1002/bdm.2141

Mahoney, J. L., Stattin, H. \& Lord, H. (2004). Unstructured youth recreation centre participation and antisocial behaviour development: Selection influences and moderating role of antisocial peers. International Journal of Behavioral Development, 28, 553-560. https://doi.org/10.1080/01650250444000270 
Malecki, C. K. \& Elliot, S. N. (2002). Children's social behaviors as predictors of academic achievement: A longitudinal analysis. School Psychology Quarterly, 17, 1-23. https://doi.org/10.1521/scpq.17.1.1.19902

Moffitt, T. E. (1993). Adolescence-limited and life-course-persistent antisocial behavior: A developmental taxonomy. Psychological Review, 100, 674-701. https://doi.org/10.1037/0033-295X.100.4.674

Monahan, K. C., Steinberg, L. \& Cauffman, E. (2009). Affiliation with antisocial peers, susceptibility to peer influence, and antisocial behavior during the transition to adulthood. Developmental Psychology, 45, 1520-1530. https://doi.org/10.1037/a0017417

Moshman, D. (2005). Adolescent psychological development: Rationality, morality, and identity, $1^{\text {st }}$ edition. Mahwah, NJ: Lawrence Erlbaum.

Plazas, E. A., Morón Cotes, M. L., Santiago, A., Sarmiento, H., Ariza López, S. E. \& Patiño, C. D. (2010). Relaciones entre iguales, conducta prosocial y género desde la educación primaria hasta la universitaria en Colombia. Universitas Psychologica, 9, 357-369. https://doi.org/10.11144/Javeriana.upsy9-2.ricp

Potocnjak, M., Berger, C. \& Tomicic, T. (2011). Una aproximación relacional a la violencia escolar entre pares en adolescentes chilenos: perspectiva adolescente de los factores intervinientes. Psykhe, 20(2), 39-52. https://doi.org/10.4067/S0718-22282011000200004

Reaves, S., McMahon, S. D., Duffy, S. N. \& Ruiz, L. (2018). The test of time: A meta-analytic review of the relation between school climate and problem behavior. Aggression and Violent Behavior, 39, 100-108. https://doi.org/10.1016/j.avb.2018.01.006

Roeser, R. W., Eccles, J. S. \& Sameroff, A. J. (2000). School as a context of early adolescents' academic and social-emotional development: A summary of research findings. The Elementary School Journal, 100, 443-471. https://doi.org/10.1086/499650

Rojas Andrade, R. \& Leiva Bahamondes, L. (2015). Psicopatología y victimización ocasional entre pares en una muestra de estudiantes chilenos. Universitas Psychologica, 14, 165-176. https://doi.org/10.11144/Javeriana.upsy14-1.pvop

Rovis, D., Jonkman, H. \& Basic, J. (2016). A multilevel analysis of adverse family relations, school bonding and risk behaviours among adolescents. Journal of Child and Family Studies, 25, 647-660. https://doi.org/10.1007/s10826-015-0223-6

Sanabria, A. M. \& Uribe Rodríguez, A. F. (2009). Conductas antisociales y delictivas en adolescentes infractores y no infractores. Pensamiento Psicológico, 6(13), 203-218. Recuperado de https://revistas.javerianacali.edu.co/index.php/pensamientopsicologico/article/view/126/374

Sanabria, A. M. \& Uribe Rodríguez, A. F. (2010). Factores psicosociales de riesgo asociados a conductas problemáticas en jóvenes infractores y no infractores. Diversitas: Perspectivas en Psicología, 6, 257-274. Recuperado de http://www.scielo.org.co/pdf/dpp/v6n2/v6n2a05.pdf

Sawyer, A. M., Borduin, C. M. \& Dopp, A. R. (2015). Long-term effects of prevention and treatment on youth antisocial behavior: A meta-analysis. Clinical Psychology Review, 42, 130-144. https://doi.org/10.1016/j.cpr.2015.06.009

Sprague, J. \& Walker, H. (2000). Early identification and intervention for youth with antisocial and violent behavior. Exceptional Children, 66(3), 367-379. https://doi.org/10.1177/001440290006600307

Stoddard, S. A., Zimmerman, M. A. \& Bauermeister, J. A. (2012). A longitudinal analysis of cumulative risks, cumulative promotive factors, and adolescent violent behavior. Journal of Research on Adolescence, 22, 542-555. https://doi.org/10.1111/j.1532-7795.2012.00786.x

Sullivan, C. J. \& Hirschfield, P. (2011). Problem behavior in the middle school years: An assessment of the social development model. Journal of Research in Crime and Delinquency, 48, 566-593. https://doi.org/10.1177/0022427810395149

Thapa, A., Cohen, J., Guffey, S. \& Higgins-D'Alessandro, A. (2013). A review of school climate research. Review of Educational Research, 83, 357-385. https://doi.org/10.3102/0034654313483907

Torres, J. \& Varela, J. (2013). El modelo de desarrollo social: la base conceptual del sistema "Communities That Care". Santiago, Chile: Fundación Paz Ciudadana.

Torres-Vallejos, J. \& Sosa, M. E. (2013). Adaptación y análisis de las propiedades psicométricas de la encuesta para jóvenes en población escolar del sistema "Communities that Care" (Tesis para obtener el grado de Licenciado en Psicología, no publicada), Facultad de Humanidades, Escuela de Psicología, Universidad de Santiago de Chile, Chile.

Varela, J. J., Sirlopú, D., Melipillán, R., Espelage, D., Green, J. \& Guzmán, J. (2019). Exploring the influence school climate on the relationship between school violence and adolescent subjective well-being. Child Indicators Research, 12(6), $2095-2110$. https://doi.org/10.1007/s12187-019-09631-9

Vitaro, F., Brendgen, M., Girard, A., Boivin, M., Dionne, G. \& Tremblay, R. E. (2015). The expression of genetic risk for aggressive and non-aggressive antisocial behavior is moderated by peer group norms. Journal of Youth and Adolescence, 44, 1379-1395. https://doi.org/10.1007/s10964-015-0296-y

Vitaro, F., Brendgen, M. \& Tremblay, R. E. (2000). Influence of deviant friends on delinquency: Searching for moderator variables. Journal of Abnormal Child Psychology, 28, 313-325. https://doi.org/10.1023/A:1005188108461

Wang, M. -T. \& Degol, J. L. (2016). School climate: A review of the construct, measurement, and impact on student outcomes. Educational Psychology Review, 28, 315-352. https://doi.org/10.1007/s10648-015-9319-1

Wilson, H. W., Stover, C. S. \& Berkowitz, S. J. (2009). The relationship between childhood violence exposure and juvenile antisocial behavior: A meta-analytic review. Journal of Child Psychology and Psychiatry, 50, 769-779. https://doi.org/10.1111/j.1469-7610.2008.01974.x

Fecha de recepción: Noviembre de 2018.

Fecha de aceptación: Mayo de 2020. 